\title{
The perception of waning signals: Decruitment in loudness and perceived size
}

\author{
ROBERT TEGHTSOONIAN and MARTHA TEGHTSOONIAN \\ Smith College, Northampton, Massachusetts \\ and \\ GEORGES CANÉVET \\ CNRS, Marseille, France
}

\begin{abstract}
When a tone or broad-band noise sweeps smoothly from a moderate intensity to a low one, the loudness at the end of the sweep is far less than what would be predicted from its intensity. The accelerated reduction in loudness, which was first reported by Canévet (1986) and confirmed in several later reports, has been called loudness decruitment, and has been tentatively interpreted as the result of some form of adaptation. Since both simple and induced adaptation have distinctive temporal profiles, we undertook a series of studies in which we varied the duration of a tone whose intensity was continuously changing, to see whether the effect of duration on decruitment resembled the effects of duration on adaptation. We discovered that the magnitude of decruitment remained unaffected when the duration of the sweep was reduced far below the durations of 90 to $180 \mathrm{sec}$ that have been used in previous studies. The same effect was observed for durations of around $20 \mathrm{sec}$, but it declined rapidly to a low level at the lowest duration of $1.0 \mathrm{sec}$. This temporal pattern is strikingly different from what has been reported for either simple or ipsilaterally induced adaptation, which suggests that neither form of adaptation can account for the entire effect. We also wanted to know whether an analogous phenomenon could exist for a sensory modality other than hearing. In the present study, observers were asked to judge the apparent size of a solid disk on a computer monitor; the disk increased or decreased continuously in area, or appeared as a series of separate areas, either in random order or in ordered progressions. We found that, as in the case of loudness, apparent size decreased more rapidly when the areas decreased continuously than would have been predicted from the actual areas themselves. We also found that some part of the accelerated shrinkage was due to a response bias in the observers' judgments that stemmed from knowledge that every value in a continuously changing series is predictably smaller (or larger, for a growing series). Whether the remaining part of the effect is a sensory phenomenon is an important issue for future research.
\end{abstract}

When a pure tone undergoes a continuous intensity sweep from a high starting level to a low ending level, its loudness shows a decrease that is much greater than would be predicted from knowledge of the intensity of the end level. That is, loudness at the end of the downsweep is much less than that of a tone presented continuously at the end level for an interval of the same duration as the sweep's. This remarkable phenomenon was reported by Canévet (1986), who showed that a $1000-\mathrm{Hz}$ tone that swept from 60 to $40 \mathrm{~dB}$ in $240 \mathrm{sec}$ underwent a 13-fold decrement in loudness, whereas a conventional loudness function (a power function with an exponent of 0.6 ) would predict only about a 4-fold drop. Canévet and

This research was initiated in the Laboratoire de Mécanique et d'Acoustique, CNRS, Marseille, France, during a visit by the first two authors in 1992 and was concluded at Smith College. We thank Sherry Shaffman for assistance in gathering the data for Experiments 3 and 4 and Bertram Scharf for helpful comments on an earlier version of this paper. Correspondence should be addressed to R. Teghtsoonian, Psychology Department, Smith College, Northampton, MA 01063 (e-mail: rtex@smith.edu).
Scharf (1990) coined the term decruitment for this unexpectedly rapid decline in loudness. In a series of experiments in which they employed the method of magnitude estimation, Canévet and Scharf demonstrated, first, that the same marked decrease in loudness occurs for a broadband noise that decreases continuously in intensity, and, second, that various judgmental biases or methodological artifacts could not account for their results. They concluded that the decruitment effect is a true sensory phenomenon. Schlauch (1992) extended these findings by showing that decruitment could be measured by a loudnessmatching procedure as well as by magnitude estimation, that the decruitment effect is limited to the stimulated ear, and that decruitment was reduced but not eliminated when attention was directed away from the auditory stimulus. He suggested that the effect is a sensory phenomenon that is, in part, mediated by a central mechanism.

Botte, Charron, and Canévet (1986), Scharf and Canévet (1989), and Canévet and Scharf (1990) developed an account of decruitment that attributes the phenomenon to adaptation, either simple or induced. Simple adaptation, the decrease in loudness of a continuously presented au- 
ditory stimulus, may account for some decruitment, but only for tones below about $40 \mathrm{~dB}$, because tones and noise above that level do not show simple adaptation. Ipsilaterally induced adaptation, the decrease in loudness in a continuous sound that is intermittently incremented in level (Canévet, Scharf, \& Botte, 1985), occurs for both tones and noise, and increases as the base level of the stimulus decreases from 40 to $20 \mathrm{~dB}$. If earlier, more intense portions of a continuously decreasing sound were to induce adaptation of later, less intense portions, and if this effect were greater below $40 \mathrm{~dB}$ where the loudness function is steeper, decruitment would occur. For noise, only this mechanism is posited; for tones, simple adaptation, where it occurs, would enhance the effect.

The duration of the intensity sweep was not systematically varied in any of these studies. Canévet (1986) used intervals ranging from 90 to $240 \mathrm{sec}$ and observed substantial decruitment throughout, but start and end intensity levels varied as well, in a nonsystematic manner. Canévet and Scharf (1990), Scharf and Canévet (1989), and Schlauch (1992) used a duration of $160 \mathrm{sec}$ in all of their studies. If decruitment is an adaptation phenomenon, we would expect it to follow a time course like that for ipsilaterally induced adaptation (if we avoid the region in which simple adaptation occurs). Scharf and Canévet (1989) showed ipsilaterally induced adaptation curves for $1000-\mathrm{Hz}$ tones at 40 and $50 \mathrm{~dB}$ SPL, with loudness decreasing about $25 \%$ in the first $20 \mathrm{sec}$ of exposure and then falling more slowly to a value of $45 \%$ at $90 \mathrm{sec}$.

Our purpose in the experiments reported here was twofold. First, we wished to confirm decruitment for 30$\mathrm{dB}$ intensity sweeps and to track it over a wider range of durations than had been previously studied, so that the size of the effect could be compared with the effects of ipsilaterally induced adaptation at those durations. Second, we wished to apply the decruitment paradigm to a different sensory modality in order to discover its generality. That is, is decruitment uniquely auditory in nature, or can it be found for other perceptual continua?

\section{EXPERIMENTS 1 AND 2 Loudness of Intensity-Varying Tones}

Two experiments were designed to study the loudness of tones following sweeps in intensity from high to low and from low to high, with the duration of the sweep as the parameter of particular interest. In Experiment 1, the subjects were older and sweep durations were from 1 to $90 \mathrm{sec}$. In Experiment 2, the subjects were younger; Experiment 2 replicated Sessions 1 and 2 of Experiment 1 , with sweep times from 10 to $90 \mathrm{sec}$.

\section{Method}

Procedure. In each session, the subject listened to 18 intensity sweeps of a $1000-\mathrm{Hz}$ tone, half from a higher to a lower $\mathrm{dB}$ level (a downsweep) and half from lower to higher (an upsweep). Each trial began with a 5 -sec reference tone that had a loudness we called 100 . The reference tone was succeeded by a 3-sec silent interval and then by the tone at the starting $\mathrm{dB}$ level for $10 \mathrm{sec}$, during which time the subject made a magnitude estimate of its loudness relative to the reference tone. The tone then swept up or down $30 \mathrm{~dB}$, at a constant rate $(\mathrm{dB} / \mathrm{sec})$ that depended on the duration of the sweep. The subject made a magnitude estimate of the sweep's loudness at the moment it ended, again relative to the reference tone. The magnitude estimates were given verbally and recorded by the experimenter. After a brief pause, the experimenter signaled the subject that another trial was to begin, and the sequence was repeated. Canévet and Scharf (1990) showed that the same measure of decruitment resulted when the subject estimated only at the beginning and end of a 180 -sec intensity sweep as when she/he estimated the changing tone every $20 \mathrm{sec}$.

In Experiment 1, the $1000-\mathrm{Hz}$ reference tone was at $60 \mathrm{~dB} \mathrm{SPL}$, and the sweep size was always $30 \mathrm{~dB}$. The three downsweep conditions were $70-40 \mathrm{~dB}, 75-45 \mathrm{~dB}$, and $80-50 \mathrm{~dB}$. In the upsweep conditions, the same three intervals were used, but went from low to high intensity. From preliminary observations, we concluded that we needed lower intensity levels in order to obtain the same magnitude of decruitment for the younger subjects, so, in Experiment 2, we reduced the reference tone and the tones bracketing the sweep by $10 \mathrm{~dB}$.

In Experiment 1, Session 1, the time that it took for the tone to sweep $30 \mathrm{~dB}$ was 30,60 , or $90 \mathrm{sec}$. Each of the six sweeps was paired with each sweep interval, for a total of 18 trials; the combinations were presented in a different random order for each subject. Because of the results, we decided to run Session 2, in which the sweep in tervals were 10,20 , and $30 \mathrm{sec}$, which were again paired with each of the possible sweeps. Session 1 was separated from Session 2 by 1 week. One year later, 6 of the 7 subjects served again in Session 3, in which sweep intervals of $1,2.5,5$, and $10 \mathrm{sec}$ were paired with each of the possible sweeps.

We ran Experiment 2 after Sessions 1 and 2 of Experiment 1 . For 5 of the 7 subjects, sweep intervals were 10,20 , and $30 \mathrm{sec}$ in Session $\mathrm{I}$ and 30,60, and $90 \mathrm{sec}$ in Session 2; for 2 subjects, the order was reversed.

Subjects. There were 7 subjects in Experiment 1. They were psychology faculty members, visitors to a psychophysical laboratory, and two of the authors. Their ages ranged from 32 to 62 years; 5 were male and 2 female. There were 7 subjects in Experiment 2. They were female students who received psychology course credit for their participation. Their ages ranged from 18 to 24 years. Except for the two authors, none of the subjects was knowledgeable about the decruitment phenomenon or the experimental design.

Apparatus. The stimuli, $1000-\mathrm{Hz}$ pure tones, were produced by a Qua-Tech waveform synthesizer (Model WSB-10C) and were managed by a Wilsonics electronic switch (Model BSIT) and a Wilsonics programmable attenuator (Model PATT). The stimuli were presented monaurally through an earphone set (TDH-49P) using MX-41 A/R cushions. The test sessions were controlled by a specialpurpose program that determined the reference tone, the silent interval, and the tone sweep that extended over the prescribed range of sound pressures and the prescribed duration. Changes occurred in steps of $0.1 \mathrm{~dB}$ at a constant rate, which depended on the duration of the sweep. The subjects were seated in an audiometric test room (Eckel Model 662D) that had a two-way viewing window. Their judgments were communicated through an intercom.

\section{Results}

The relation between the intensity of tones and their loudnesses in Sessions 1, 2, and 3, plotted separately for the start and the end of a sweep but averaged over sweep times, is shown in Figure 1. (All averages are geometric means.) The open symbols, which show loudness at the beginning of a sweep, should correspond to standard loud- 


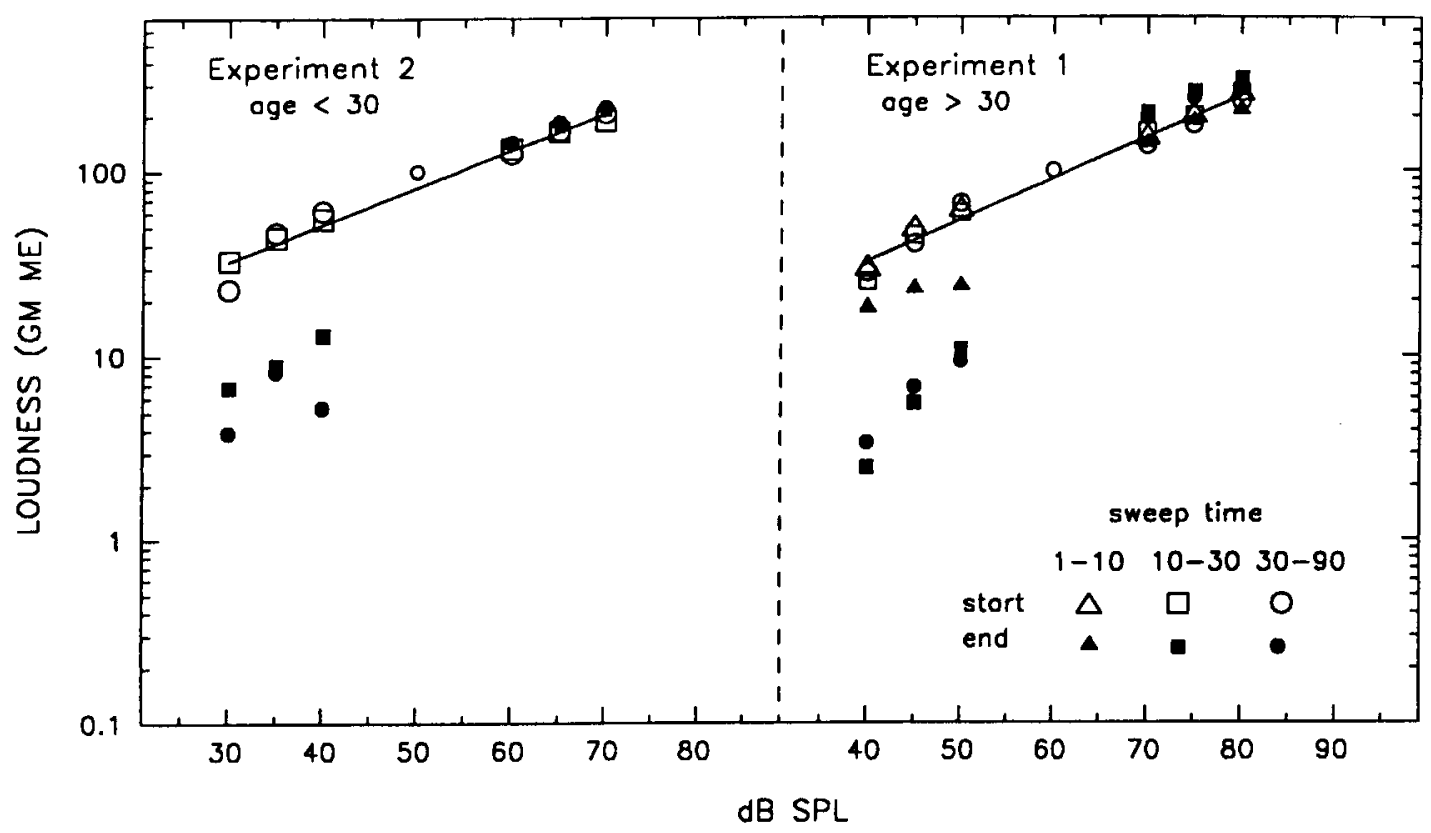

Figure 1. Loudness estimates of the start and end levels of a tone that swept, either up or down, over three 30-dB ranges at different sweep times, as a function of the sound pressure level at which the judgment was made. Judgments were averaged (geometric means) over 1-10 sec, 10-30 sec, and 30-90 sec sweep times. The left-hand panel shows results for a group of younger subjects (for sweep times $10-90 \mathrm{sec}$ ); the right-hand panel, older subjects (for sweep times $1-90 \mathrm{sec}$ ). Unfilled symbols show judgments made at the start of a sweep; filled symbols, the end of a sweep. Thus, in the right-hand panel, the unfilled circle at $(40 \mathrm{~dB}, 22)$ shows the mean loudness estimate of 22 for a 40 -dB tone at the start of upsweeps that lasted 30,60 , or $90 \mathrm{sec}$, whereas the filled circle at $(40 \mathrm{~dB}, 3.3)$ shows the mean loudness estimate of the same tone at the end of comparable downsweeps. Similarly, the unfilled square at $(\mathbf{7 0} \mathrm{dB}, 180)$ shows the mean loudness estimate of a 70-dB tone at the start of downsweeps that lasted 10, 20, or 30 sec, whereas the filled square at $(70 \mathrm{~dB}, 210)$ shows the mean loudness estimate of the same tone at the end of comparable upsweeps. The small unfilled circles at $(50 \mathrm{~dB}, 100)$ and $(60 \mathrm{~dB}, 100)$ represent the reference tones, assigned the value 100, for younger and older subjects, respectively. The straight lines are the best-fitting power functions to the judgments made at the start of a sweep.

ness functions measured by magnitude estimation. For subjects under 30 , the exponent of the fitted power function is 0.4 ; for those over 30, it is 0.5. (Despite the often cited exponent of 0.6 for sound pressure, values from 0.4 to 0.5 for magnitude estimation are frequently reported in the literature; see, e.g., the insert in Figure 1 of Canévet, Hellman, \& Scharf, 1986.) The order of sessions does not appear to have had a systematic effect on those judgments.

The filled symbols show loudness at the end of a sweep. Those falling below the loudness function indicate a sizable decruitment effect for sweep times from 10 to $90 \mathrm{sec}$ for both groups; the effect was greatly diminished, but not absent, at the very short sweep times in Experiment 1 . For the older subjects, there is an apparent relation between the location of the sweep on the intensity scale and the amount of decruitment-the lower the location, the greater the effect. In the region where duration has no effect, from 30 to $90 \mathrm{sec}, \mathrm{a} 40-\mathrm{dB}$ tone at the end of a sweep is judged to be about $10 \%$ as loud as when it occurs at the start; a $45-\mathrm{dB}$ tone, $12.5 \%$ as loud; a $50-\mathrm{dB}$ tone, $18.3 \%$ as loud. However, for the younger subjects, the amount of decruitment may have been somewhat reduced and less clearly related to the location of the sweep: The comparable figures for sweeps ending at 30,35 , and $40 \mathrm{~dB}$ are $21 \%, 21 \%$, and $24 \%$, respectively.

The data also suggest an enhancement of loudness for upsweeps at times from 10 to $90 \mathrm{sec}$. These smaller effects are not easy to see on a logarithmic scale. For the older subjects, the size of the effect is on the average about $30 \%$ (i.e., 70,75 , and $80 \mathrm{~dB}$ SPL sound about $30 \%$ louder when they end a sweep than when they begin it). The younger subjects also show enhancement, but the effect is much smaller, on the order of $10 \%$. Schlauch (1992) also measured such an enhancement on an upsweep.

The data may also be plotted as in Figure 2, in which loudness is shown as a function of sweep time, with the $\mathrm{dB}$ level of the tone, start or end, as a parameter; this allows us to look at the time course of decruitment. The open symbols labeled "start" show loudnesses for tones at the start of a sweep, and those labeled "end" show loudnesses for the same tones at the end of a sweep, for each of the six $\mathrm{dB}$ levels, whereas the filled symbols are averages (geometric means) over sound pressure levels. When the same sweep time occurred in different sessions $(10 \mathrm{sec}$ and $30 \mathrm{sec})$, the data were combined over the two sessions. 
Friedman analyses of variance by ranks were performed on the geometric means of loudness judgments of 30,35 , and $40 \mathrm{~dB}$ (younger subjects) and of 40,45 , and $50 \mathrm{~dB}$ (older subjects) when those intensities ended a sweep for each sweep time. For the younger subjects, the analysis (for 7 subjects and five sweep times) did not allow rejection of the null hypothesis $\left(F_{r}=2.86\right.$ is distributed as $\chi^{2}$ with $d f=4$, not reliable at the .05 level). The associated Kendall coefficient of concordance is $W=.102$. The results for downsweeps at times from 10 to $90 \mathrm{sec}$ are essentially identical; the amount of decruitment does not change over a ninefold change in sweep time.

For the older subjects, the Friedman analysis (for 6 subjects who served in all three sessions, at eight sweep times) allowed the rejection of the null hypothesis at the .05 level of significance $\left(\chi^{2}=28.89, d f=7\right)$; the associated Kendall coefficient of concordance is $W=0.684$. Subsequent pairwise comparisons ( $\alpha=.05$, one-tailed test) showed that a sweep time of $1 \mathrm{sec}$ differed from sweep times of $10,20,30,60$, and $90 \mathrm{sec}$ and that $2.5 \mathrm{sec}$ differed from $20 \mathrm{sec}$, but that no other sweep times differed from each other. (Kendall coefficients of concordance $W$ were calculated separately for the three sets of sweep times, 1-10, $10-30$, and 30-60 sec, and were $0.878,0.299$, and 0.049, respectively; only the first reached significance at the .05 level.) Thus, for these subjects, the inclusion of even shorter sweep times down to $1 \mathrm{sec}$ revealed an effect: The curve falls rapidly and appears to reach an asymptote around $20 \mathrm{sec}$.
The enhancement effect on upsweeps was also revealed to be time dependent. A Friedman analysis of variance by ranks of the geometric means of loudness judgments for 70,75, and $80 \mathrm{~dB}$ when they ended an upsweep (for six subjects at eight sweep times) indicated reliable differences $\left(F_{r}=23.54\right.$, distributed as $\chi^{2}$ with $d f=7$, $p<.05$ ). The associated Kendall coefficient of concordance was $W=0.561$. Thus, below about $10 \mathrm{sec}$, end loudness was depressed, and asymptotic enhancement was reached at about $20 \mathrm{sec}$.

\section{Discussion}

Our studies provide yet another confirmation of Canévet's (1986) original finding of decruitment. The phenomenon is robust, which is evident not only in the averaged data but also in the results from each of the 14 subjects, although the size of the effect varied considerably. Among the older subjects, one consistently showed loudness decreases of over $95 \%$, whereas another never exceeded $75 \%$ and averaged $55 \%$; among the younger subjects, one consistently showed loudness decreases over $90 \%$, whereas another never exceeded $89 \%$ and averaged $47 \%$. We could speculate that some of the variability was related to differences in individual thresholds; it is apparent now that threshold data would be valuable in interpreting changes of loudness in intensity-variable tones.

Our results do not support the idea that decruitment depends solely on loudness adaptation; there was little or no simple adaptation at the sound pressure levels we

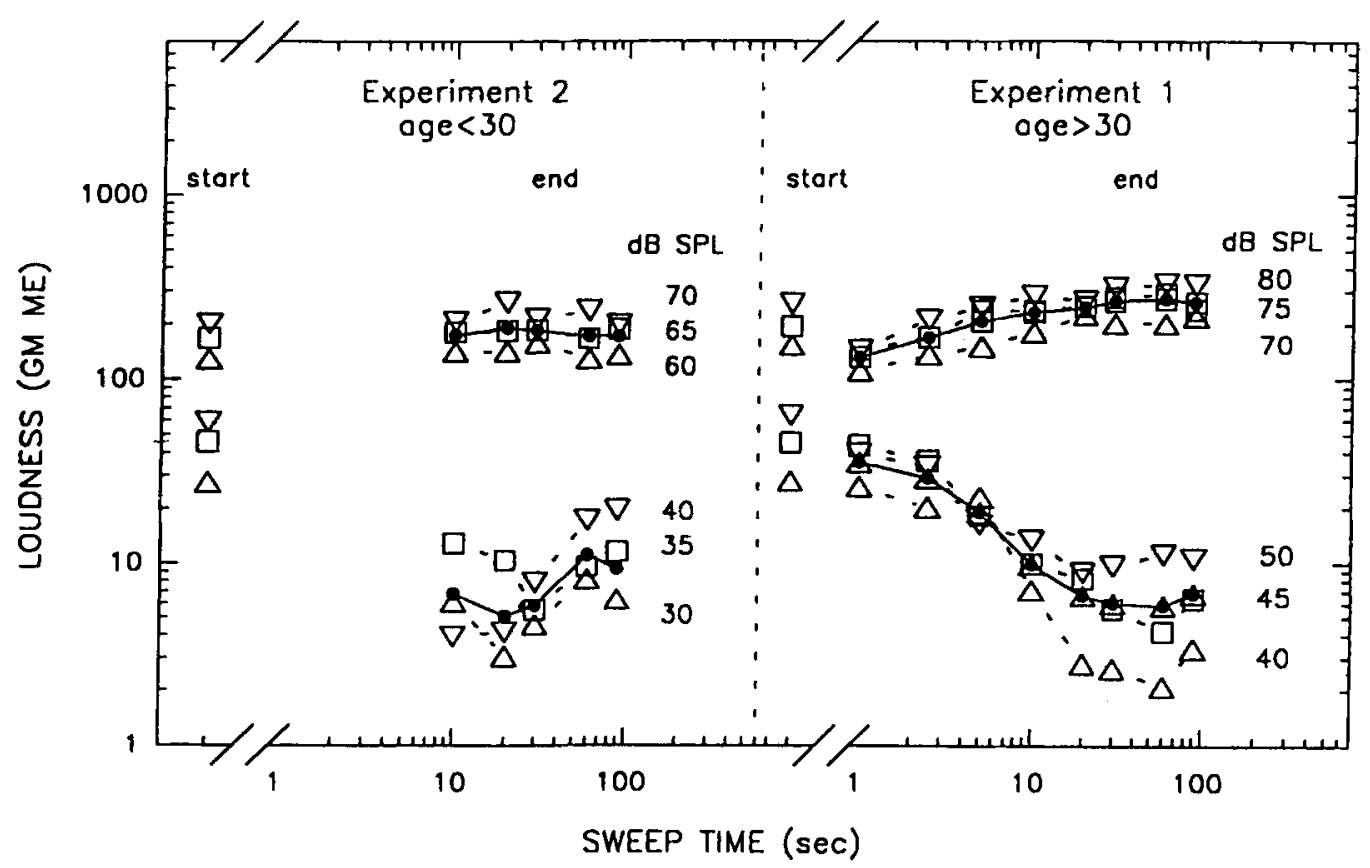

Figure 2. Loudness estimates (geometric means) of the end level of a tone that swept, either up or down, over three 30-dB ranges, as a function of sweep time. The left-hand panel shows results for a group of younger subjects; the right-hand panel, older subjects. The unfilled symbols show loudness estimates for each sweep range; the filled symbols, averages (geometric means) over ranges. For comparison, at the left of each panel are judgments made at the start of a sweep. 


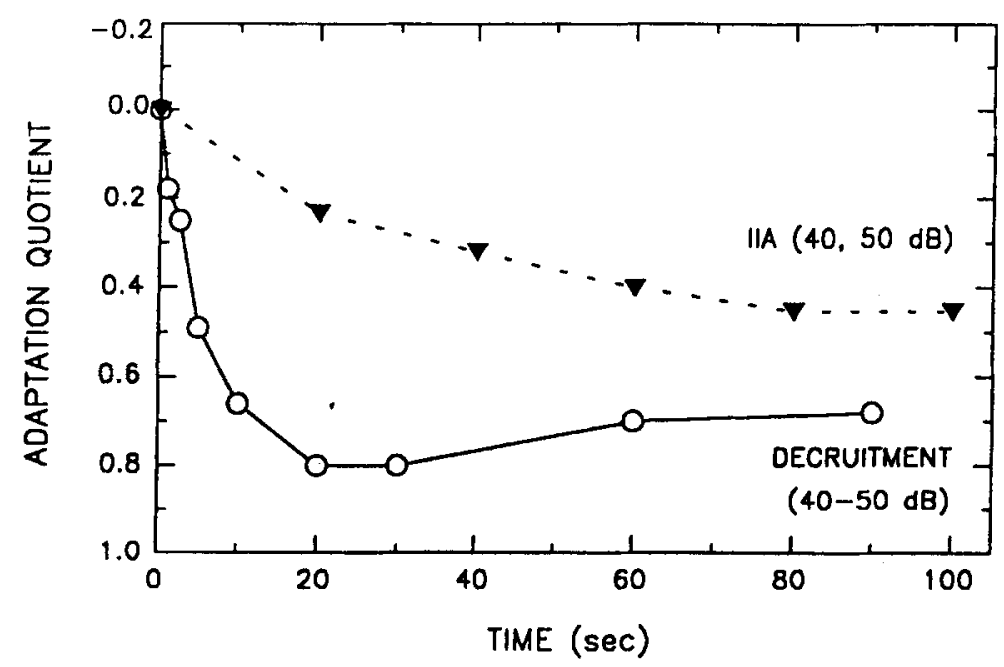

Figure 3. Time course of loudness changes in two paradigms. Loudness changes are expressed as adaptation quotients, $\mathrm{AQ}=\left(E_{\mathrm{i}}-E_{t}\right) / E_{\mathrm{i}}$, where $E_{\mathrm{i}}$ is the initial judgment and $E_{t}$ is the judgment at time $t$, calculated for each subject and averaged. The filled triangles show ipsilaterally induced adaptation data, averaged for tones of 40 and $50 \mathrm{~dB}$, from Scharf and Canévet (1989). The unfilled circles show our decruitment data for older subjects averaged over end levels of 40, 45, and $50 \mathrm{~dB}$.

used. Ipsilaterally induced adaptation certainly occurs for $40-$ to $50-\mathrm{dB}$ SPL $1000-\mathrm{Hz}$ tones, as demonstrated by Scharf and Canévet (1989), but its time course is rather different from that for decruitment. In Figure 3, we compare Scharf and Canévet's ipsilaterally induced adaptation curve with our decruitment curve for older subjects, averaged over the three end levels of 40,45 , and $50 \mathrm{~dB}$ SPL. Scharf and Canévet presented their adaptation data expressed as mean adaptation quotients, and we converted our data (using their procedure) to facilitate comparison. The adaptation quotient, $\mathrm{AQ}$, is defined as $\left(E_{i}-E_{t}\right) / E_{i}$, where $E_{i}$ is the initial judgment and $E_{t}$ is the judgment at time $t$, calculated for each subject and arithmetically averaged. An AQ of 0 means there was no change in loudness due to adaptation or decruitment, whereas an $A Q$ of 1 means the sound became inaudible. Given the marked difference between the adaptation curve and the decruitment curve, it seemed unlikely that adaptation could account for the entire decruitment effect.

It should be noted that the interpretation of the effects of varying sweep time is complicated by the confounding of sweep time with the rate of change in SPL. Because all sweeps covered a constant span of $30 \mathrm{~dB}$, shorter sweep times necessarily entailed faster rates of change. The effect of rate of change requires independent evaluation through a procedure in which sweep time is held constant, but to do so would confound rate of change with sweep span and the start (or end) point in the sweep.

Our data add to the evidence of an "upcruitment" (recruitment has been preempted for use in a different context) phenomenon that is associated with signals sweeping upward in intensity, but it appears to be more complicated than previously has been realized. At short inter- vals (1-20 sec), loudness was depressed below its expected level on the upsweep, whereas at longer intervals, loudness was enhanced. We do not know what to make of the fact that $20 \mathrm{sec}$ was also the point at which decruitment stabilized, but the coincidence should be noted.

\section{EXPERIMENT 3 Apparent Size of Discs Continuously Varying in Diameter}

We know of no demonstrations of a decruitment-like phenomenon in other sensory modalities. Should they be found, the possibility that decruitment is a supramodal phenomenon is raised, and an explanation of loudness decruitment as adaptation becomes less plausible. In the next two experiments, we examined the apparent size of a disk that continuously increased or decreased in area.

In this initial investigation of "size decruitment," we followed the design that Canévet and Scharf (1990) employed to investigate loudness decruitment in their Experiment 1. Their stimulus (1) continuously increased, (2) continuously decreased, with loudness judgments made every $20 \mathrm{sec}$, or (3) was presented at discrete values in random order, with a judgment made after each presentation. In the present experiment, the stimulus was a red disk on a gray background presented by computer on a video monitor; the disk could change continuously in area, increasing or decreasing, or it could be displayed at constant values varying in size in any designated order.

\section{Method}

Subjects. There were 11 subjects, all female undergraduate students, who received psychology course credit for their participation. 
Stimuli. The stimulus was a solid red disk on a dark gray background in the center of a video monitor $(205 \mathrm{~mm}$ high and $275 \mathrm{~mm}$ wide), viewed at light levels typical of an office environment. The disk could increase or decrease in radius one pixel at a time. In an increasing continuous condition, the disk was first presented at a diameter of $18 \mathrm{~mm}$, then increased to a diameter of $185 \mathrm{~mm}$ in $150 \mathrm{sec}$; in a decreasing continuous condition, the disk shrank from 185 to $18 \mathrm{~mm}$ in $150 \mathrm{sec}$. At the beginning, and at $15-\mathrm{sec}$ intervals thereafter, a beep signaled the subject to make a judgment. In a random discrete condition, the disk was presented at the 11 areas at which judgments were made in the two continuous conditions, but the areas occurred in random order; a new area appeared every $15 \mathrm{sec}$ and disappeared when the subject made her judgment. The subjects were seated so that displays were approximately at eye level, at a distance of 550-600 mm.

Procedure. Each subject was tested in three conditions: increasing continuous (IC), decreasing continuous (DC), and random discrete (RD). The subjects performed in Conditions IC and DC first (in alternating order from subject to subject), then in RD, then in IC and DC in the opposite order, and finally in RD. Each subject was instructed to assign a number to represent the two-dimensional extent of the disk as it appeared to her at each occurrence of the signal. She was informed before each condition of the nature of the change (increasing, decreasing, or random). Each subject entered her judgments on a computer keyboard.

\section{Results}

Although all 11 subjects completed the entire procedure, the second judgments for the first 5 subjects were not collected, owing to a programming error. (The picture is further complicated by the fact that 1 subject did not produce usable data in the Random condition.) Rather than discard these data, we have treated them separately. Geometric mean responses were calculated and are shown in the left panel of Figure 4. Data from the remaining 6 subjects, for whom both sets of judgments in each condition are available, were treated in the same way; the means are shown in the right panel of Figure 4. Because these sample sizes were small, no statistical tests of significance were attempted; but all of the critical comparisons occurred again in Experiment 4, in which such tests were done, and the results are reported below.

The size data do not show such marked nonlinearity in $\log -\log$ coordinates as the Canévet-Scharf loudness data do. Even though there is some curvature in the data for smaller disks, we thought it not so severe as to preclude the fitting of a straight line. The resulting power functions are good fits in most cases, and the exponent (measured as the slope of the linear fit) serves as a convenient index of the degree to which decruitment may have occurred: An accelerated reduction in loudness will yield a function with a larger exponent. Thus, our use of this measure is not intended to imply a judgment about the shape of the perceived size functions; the power function exponent is used here as a convenient index of treatment effects.

In Figure 4, both sets of data indicate the existence of a "decruitment" phenomenon for apparent size. The exponent for the decreasing condition is the highest of the three, 1.05 for both groups; the exponent for the increasing condition is intermediate, .80 and .79 ; the exponent for the random condition is lowest, .75 and .69. Mean judgments for the largest stimulus are about the same in the decreasing and random conditions, whereas the smallest stimulus was judged to be about one quarter as large when it ended a downsweep as when it was part of a ran-

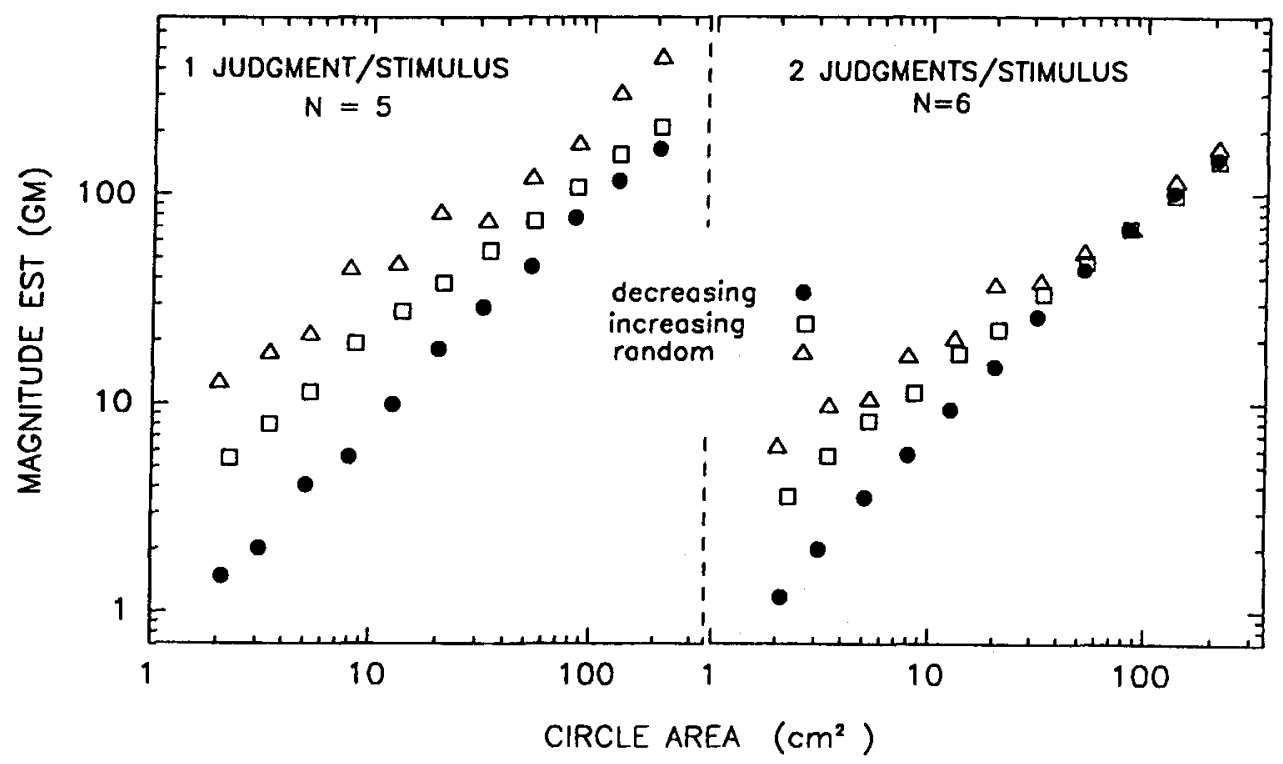

Figure 4. Size judgments of a disk that is continuously decreasing in area, continuously increasing in area, or occurring at discrete intervals with area randomly chosen. The left panel shows data from 5 subjects judging once per stimulus; the right panel, from 6 subjects judging twice per stimulus. (The areas shown for different conditions differ slightly. This reflects the operation of the program, which recorded the actual area at the time of the signal calling for a judgment.) 
dom sequence. It is also noteworthy that the subjects in the decreasing condition were much more likely to give judgments of less than 1.00 .

However, it is more difficult to interpret the results of the increasing condition. Because subjects were free to determine the scale factor for their judgments, there was a risk that with such a small sample the mean judgment of the smallest area might differ from the judgment of that area as part of the random condition, and this is what happened. Although the slopes of the two functions are in the expected direction for an "upcruitment" effect similar to that obtained in our (and other) studies of loudness, all of the judgments in the increasing condition are at or below the corresponding values in the random condition; and it is therefore difficult to speak of size enhancement for these data. This problem of a lack of direct comparability across conditions was addressed in Experiment 4 .

An examination of the data summarized in Figure 4 led us to consider the possibility that, in the continuous conditions, simply knowing that the stimulus would change in only one direction, growing either smaller or larger, could produce a larger exponent than that obtained in the random condition. Since knowing the direction of change makes it extremely unlikely that judgments for successive areas will show the kind of reversal that is common when stimuli are randomly ordered, we expected a growing bias toward underestimation (in the decreasing condition) and overestimation (in the increasing condition). This hypothesis was explicitly addressed in Experiment 4.

These considerations led to another experiment with the following features. First, we included two new conditions, decreasing discrete and increasing discrete, to allow separate evaluation of continuity of change as opposed to knowledge of the direction of change. Second, we used two groups of 10 subjects each, one judging only decreasing and random conditions, the other only increasing and random conditions. Third, in order to ensure greater comparability across conditions, we included in all conditions a designated standard that had an assigned modulus. In the decreasing conditions, the initial display was assigned the value of 100; in the increasing conditions, the value 1 ; in the random conditions the initial stimulus was either the largest disk with an assigned value of 100 , or the smallest disk with an assigned value of 1 .

\section{EXPERIMENT 4 Apparent Size of Disks Continuously or Discretely Varying in Diameter}

\section{Method}

Subjects. There were 40 subjects, all female undergraduates who received psychology course credit for their participation; none had served in Experiment 3.

Stimuli. The stimuli were identical to those in Experiment 3.

Procedure. There were six conditions, increasing continuous and increasing discrete, decreasing continuous and decreasing discrete, and random low and random high. In the increasing conditions, the size of the disk increased montonically from the initial stimulusthe smallest disk, with a designated modulus of 1 -to the largest disk. In the decreasing conditions, the size of the disk decreased monotonically from the initial stimulus - the largest disk, with a designated modulus of 100 - to the smallest disk. The continuous conditions were identical to those in Experiment 3, with the disk on the screen at all times, visibly expanding or contracting. In the discrete conditions, the screen was blank except when a disk, which was either the smallest, designated 1, followed by successively larger ones, or the largest, designated 100 , followed by successively smaller ones, was presented. In the random conditions, the first stimulus was either the smallest, designated 1 (random low), or the largest, designated 100 (random high); the other sizes were presented in random order. In both the discrete and random conditions, stimulus presentation was terminated when the subject responded.

Ten subjects judged in both of the increasing conditions and in the random low condition; 10 subjects judged in both of the decreasing conditions and in the random high condition. For a given subject, the same modulus was always assigned to the same stimulus. (The data for 1 subject in the decreasing group were excluded because of her failure to understand the instructions.) The continuous and discrete conditions were counterbalanced for order within increasing and decreasing conditions; the random condition was always presented third. Each subject was instructed about the nature of a condition prior to making her judgments. One set of judgments was obtained from each subject for each of the three conditions in which she served.

Because the primary purpose of this study was the comparison of the continuous and discrete conditions, the two varieties of each condition always preceded the random condition in order to avoid order effects due to judging the random condition first. The latter was included in the hope that the results would be the same for the two varieties - high and low. However, in view of the evidence for order effects found in Experiment 3, we decided a priori that, if the random high and low results differed, we would discard them and replace them with data gathered from new, independent groups of subjects. In fact, this was the case. The exponent for random low was greater than that for random high $[0.83$ vs. $0.58, t(17)=3.54$, $p<.05]$. Accordingly, two new groups of 10 subjects each were tested, one for random high, the other for random low; the exponents did not differ [ 0.56 for random high, 0.64 for random low, $t(18)<$ 1.0]. These data are reported below for all analyses involving random conditions.

\section{Results}

Decreasing condition. The left panel of Figure 5 shows the geometric mean magnitude estimates of apparent size under the three conditions. A comparison of the continuous with the random conditions confirms the finding in Experiment 3 that there was a decruitment effect: At the end of the sweep the judged size in the continuous condition is an order of magnitude smaller than that for the same area in the random condition. The index of that effect, the slopes of those two functions, are 1.12 and 0.56 , respectively $[t(17)=3.67, p<.05]$. The second question, and the focus of this experiment, is whether the discrete decreasing condition yielded judgments that were progressively smaller than those obtained for corresponding areas in the random high condition. The steeper slope for the discrete decreasing condition $(0.78)$ is significantly greater than that for the random condition $[0.56 ; t(17)=2.36, p<.05]$. Knowledge of the direction of change did contribute to the decruitment effect. A third question is whether there was a decruitment effect beyond what is accounted for by the knowledge bias: Will the slope for the continuous condition be significantly greater than that for the discrete decreasing condition? 


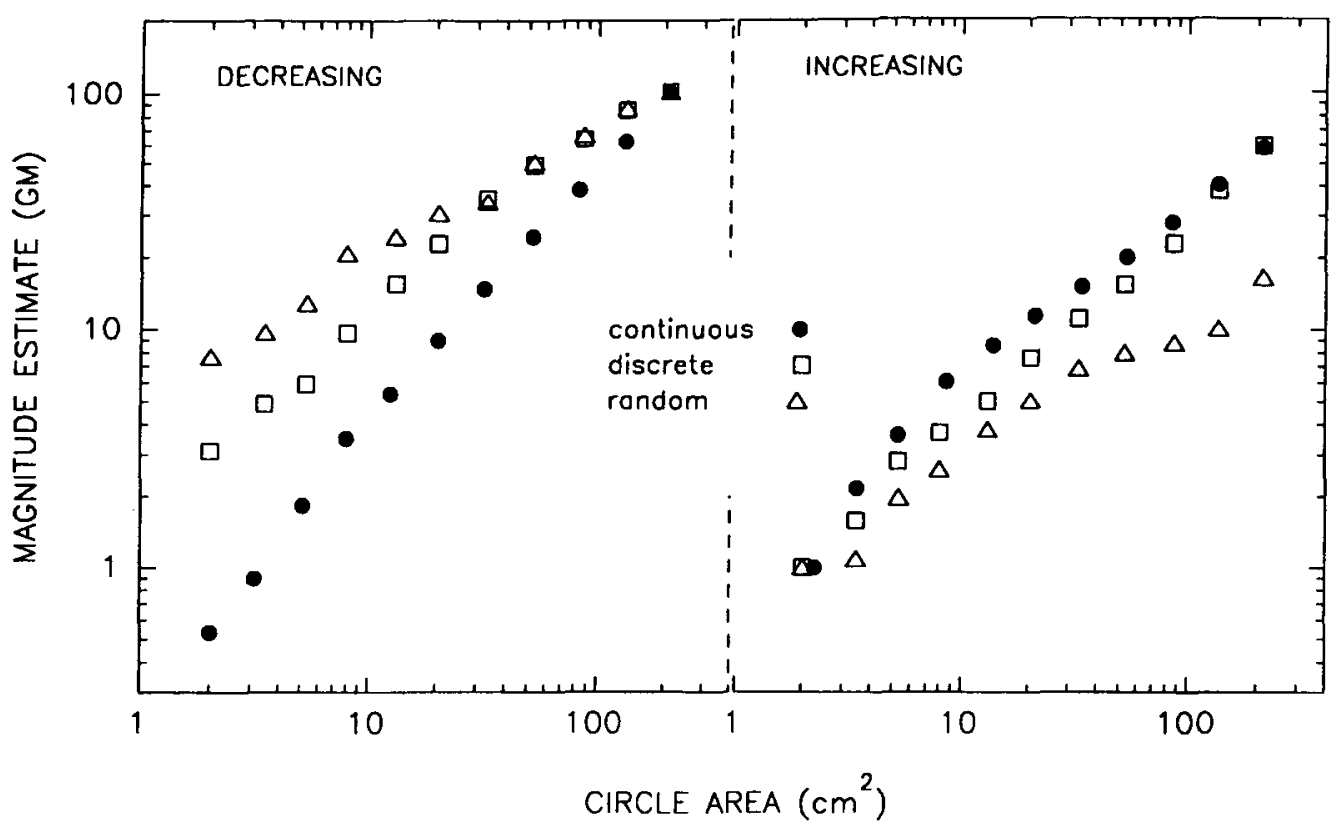

Figure 5. Size judgments of a disk that is continuously changed in area in a single direction, discretely changed in area in a single direction, or discretely changed with area randomly chosen. The left panel shows judgments when the disk was decreasing (or random); the right panel, when it was increasing (or random). One group of subjects is represented in the left panel; another group, in the right. A third group supplied the random independent judgments shown in the right-hand panel. (See note at the end of the Figure 4 caption.)

As can be seen in Figure 5, the answer is yes $[t(8)=3.67$, $p<.05]$.

Increasing condition. The right-hand panel of Figure 5 shows the geometric mean magnitude estimates of apparent size under the three conditions. As in Experiment 3, the function for the continuous condition ( 0.82$)$ is steeper

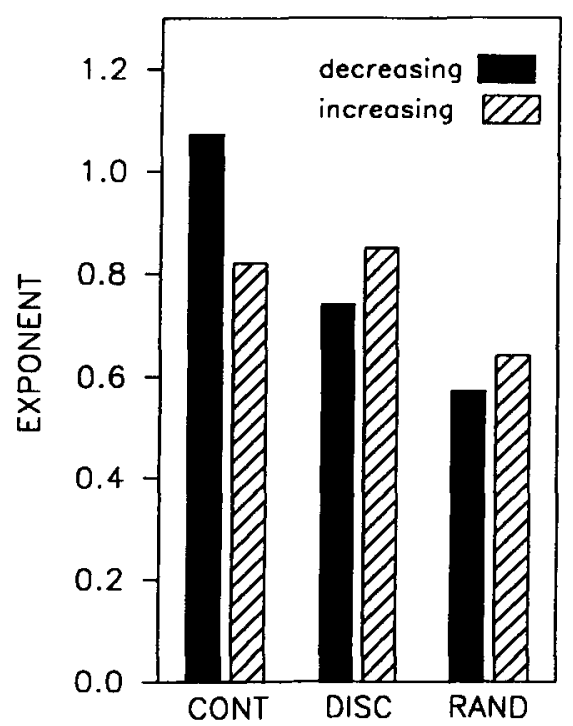

Figure 6. Apparent-size exponents for a disk changed continuously in area in a single direction (CONT), discretely changed in area in a single direction (DISC), or discretely changed with area randomly chosen (RAND). than that for the random low condition (0.64), but in this case, with the functions aligned at the smallest area, the progressive enhancement of perceived size is apparent. Is there evidence for an effect due to knowledge of the direction of change? As in the case of the decreasing conditions, the slope for the discrete condition $(0.85)$ is steeper than that for the random condition $[t(18)=2.96, p<.05]$, showing virtually the same degree of accelerated growth as that for the continuous condition. Indeed, there is no reliable difference between those conditions $[t(9)=1.32$, $p>.05$ ], which indicates that all of the enhancement that was observed in the continuous increasing condition is attributable to knowledge of the direction of change. It should be noted, however, that subjects assigned larger numbers to all stimuli (except end points) in the continuous than in the discrete condition. There is a suggestion, then, that the enhancement found for loudness also occurred for apparent size, but, if it did, it was much smaller than the size reduction associated with continuous decrease.

Figure 6 summarizes these data, showing exponents for each of the six conditions.

\section{Discussion}

The existence of a hitherto undetected effect on the apparent size of a visually presented form that decreases continuously in size is strongly suggested by these data, and as a convenient phrase for reference purposes we propose accelerated shrinkage. Although part of the decrease in magnitude estimates of size is attributable to the subject's knowledge that the change was unidirectional, there remains a substantial part that was independent of 
this knowledge. There is a difference in exponent of 0.5 between random presentation of stimuli that varied in area and presentation of stimuli that continuously decreased in area; one-third of the difference in exponent is attributable to the knowledge of the direction of change and two thirds to the size sweep itself.

Canévet and Scharf (1990) conducted an analogous experiment with tones. In one condition, the tone was presented as burst pairs at levels from 30 to $70 \mathrm{~dB}$ in random order (similar to our random conditions); in three other conditions, the tone was presented discretely (with duty cycles of $0.5,0.1$, and 0.05 , respectively) but decreased monotonically from 70 to $30 \mathrm{~dB}$ in $160 \mathrm{sec}$ (similar to our decreasing discrete condition); in a fifth condition, the tone decreased continuously from 70 to $30 \mathrm{~dB}$ in $160 \mathrm{sec}$ (similar to our decreasing continuous condition). Their results were very much like the present ones: The discretely decreasing tones were judged to be less loud than the randomly presented tones but louder than the continuously decreasing tones at levels below $50 \mathrm{~dB}$. Canévet and Scharf argued that, since making a tone intermittent decreases simple adaptation, and since simple adaptation may be involved in decruitment under $40 \mathrm{~dB}$, the smaller effect for interrupted tones was adaptation based. Because our results for size did not depend on adaptation (as far as we know, no evidence for "size adaptation" has been reported), we suggest that both Canévet and Scharf's results, as well as ours, depend on the knowledge that allows the subject to avoid reversals in judgment. Schlauch (1992) also collected data for decreasing continuous and intermittent (duty cycle 0.5 ) tones, and these findings also showed a more rapid decline in loudness for the continuous condition, although the difference was not as great as in Canévet and Scharf's study. Schlauch also evaluated decruitment with a loudness-balancing task. A monaurally presented reference tone was decreased continuously over a range of $40 \mathrm{~dB}$ in $180 \mathrm{sec}$; following its offset, a comparison tone was presented to the contralateral ear and was adjusted by the observer to match the loudness of the reference tone at the end of its sweep. He measured a smaller decruitment effect with loudness balancing than with magnitude estimation and attributed the difference to attentional factors. Our results suggest that the difference between his results for the two methods may have been due, in part, to knowledge of the direction of change. This could have been operating in his magnitude estimation task, in which subjects made a series of judgments as the tone decreased, but not in the loudness-balancing task, in which subjects made only a single judgment, which was also at the end of the sweep.

The knowledge factor may play an even larger role in accounting for changes in perceived magnitude in increasing conditions. It is worth recalling that most, if not all, of the size enhancement observed with continuously increasing disks was attributable to predictability in the direction of change (the function for the increasing discrete condition was very close to that for the increasing continuous condition), and the same may be true of the loudness enhancement in previous studies. But our finding of enhanced loudness when sound pressure increases continuously is based on a single judgment obtained at the end of the sweep, and is therefore unlikely to reflect a bias due to knowledge about direction of change.

Phenomenally, loudness decruitment is far more compelling than the change in apparent size that we measured. A listener often feels that the tone at the end of a downsweep has almost disappeared, and an occasional judgment of 0 does occur. A viewer of a decreasing disk has no such experience; the first two authors, who served as observers, were surprised to find the decrement in apparent size shown in their data. It is possible that, if the unchanging frame of reference provided by the monitor could be eliminated, the size effect would become more apparent. Furthermore, a more marked effect might have been obtained if the changes in radius could have been made more smoothly. The one-pixel step in radius change was readily discernible and did not capture the same quality of seamless change that characterized the sweeps in sound pressure levels. However, in Experiment 4, the disk at the end of a downsweep in the continuous condition was judged to be $8.5 \%$ the size of that same disk in the random condition and $16 \%$ the size of the disk in the discrete condition. This may be compared to the $1000-\mathrm{Hz}$ tone judged as $9 \%-15 \%$ as loud at the end as at the beginning of a downsweep.

Some consideration should be given to the possibility that loudness decruitment and accelerated shrinkage for disks are the outcomes of some higher level process rather than truly sensory in nature. Such a conjecture would become increasingly attractive if similar phenomena were to be found for additional intensive continua. Indeed, Schlauch (1992) raised the possibility that some part of the phenomenon could be traceable to central factors. However, it should be recalled that Schlauch tested for transfer of decruitment to the contralateral ear and, using repeated magnitude estimates, found none, which strongly implied that a sensory effect occurred in the training ear (see also Schlauch \& Morison, 1995). It would be of great interest to do an analogous test for size judgment by presenting a shrinking disk to one side of the visual field and testing in the other.

It is also worth noting that, for both loudness and apparent-size studies, our procedures featured a fixed value for a parameter that may have exerted an influence on the outcome. In each case, the physical parameter (sound-pressure level in one case, area in the other) was changed over time (in continuous conditions) at a constant rate on a logarithmic scale; that is, in both cases the physical parameter was changed by a fixed ratio per unit time. All of the research in the decruitment literature has been done in this way, and we saw no reason to depart from this convention in the present research. But two facts about this procedure must be borne in mind. First, neither of the corresponding perceptual attributes, loudness or perceived size, will be perceived as changing at a constant 
rate. Assuming that a power function describes the relation between the physical parameter and the corresponding perceptual attribute, changes in the first at a constant velocity on a logarithmic scale will yield changes in the second of the same nature. In short, the traditional procedure for the study of decruitment produces a distinctly nonlinear change in loudness, and a similar statement can be made about the perceived size studies. The second fact is that the rate of change in the physical parameter can be adjusted to suit the needs of the experimenter (see Marks \& Slawson, 1966), and in particular, it is possible to adjust the rate at which the perceptual attribute (loudness or perceived size) changes over time. That function could be linear, or it could take any of a number of nonlinear forms. Our research shows that, for both disks and tones, continuous reduction of the physical parameter at a constant logarithmic rate results in an accelerated reduction in the corresponding perceptual attribute. But it remains for future research to determine to what degree that effect is sensitive to the way in which the perceptual attribute changes over time.

Finally, it should be noted that since this paper was first submitted for publication, there has appeared a report (Neuhoff, 1998) of research employing acoustic signals that increased or decreased continuously, covering a 15-dB range in $1.8 \mathrm{sec}$. Listeners were asked to judge the amount of the change in loudness on a 5-point category scale and consistently reported that the upsweeps covered a larger range than did the downsweeps. This seems inconsistent with the results of the present research and the earlier literature on decruitment: Because the size of the decruitment effects has been at least as large as, or larger than, the corresponding "upcruitment" effects, it is apparent that the range between judgments of loudness at the start and again at the end of a downsweep are as large as, or larger than, they are in the case of an upsweep, a finding that seems in direct conflict with Neuhoff's report. However, it must be borne in mind that his listeners made no direct judgment of loudness per se, but were asked to judge change in loudness. Another procedural difference from the present study lies in the fact that our subjects had signals that all began with a 10 -sec plateau during which they could form a judgment of the initial loudness level; Newhoff included no such plateau in his procedure. It is not obvious how such procedural differences could account for the seeming disparity between his findings and ours; it remains a problem for further study.

\section{SUMMARY AND CONCLUSIONS}

The first two experiments provide further validation for the phenomenon of loudness decruitment: A tone continuously decreasing in amplitude from a moderate starting point reaches a loudness substantially lower than what that amplitude would yield either alone or at the end of a series of decreasing but separate amplitudes. We have also shown that the effect is unchanged by reducing the duration of the downward sweep well below previously reported values. Only when the sweep time is shorter than 10-20 sec does the magnitude of decruitment begin to diminish, and even at $2.5 \mathrm{sec}$ there is still an effect. This relation to duration resembles neither that for simple adaptation nor that for ipsilaterally induced adaptation. The lack of correspondence makes these mechanisms unlikely as factors in decruitment. However, Canévet (1998) has recently reported evidence for what he calls "medium-term" adaptation, operating over a range of durations less than $10 \mathrm{sec}$; it is possible that medium-term adaptation is implicated in decruitment.

We have also demonstrated, for a quite different perceptual continuum, that a continuously decreasing stimulus - in our case, a shrinking disk -is associated with a change that is much more rapid than expected - in our case, a marked decrease in apparent size. The nature of the mechanisms underlying the effect remains obscure, but in the absence of any evidence for a process that is analogous to loudness adaptation, some other explanation must be sought.

\section{REFERENCES}

Botte, M. -C., Charron, S., \& Canévet, G. (1986). On the measurement of loudness adaptation. In B. Berglund, U. Berglund, \& R. Teghtsoonian (Eds.), Fechner Day '86 (pp. 53-58). Stockholm: International Society for Psychophysics.

CANÉvET, G. (1986). Estimation de sonie pour des sons purs à variation monotone de niveau. Acustica, 61, 256-264.

CANÉVET, G. (1998). The time course of lateralization threshold for a monaural increment in a diotic pure-fone pedestal. Manuscript submitted for publication.

Canévet, G., Hellman, R., \& Scharf, B. ( 1986). Group estimation of loudness in sound fields. Acustica, 60, 277-282.

Canévet, G., \& Scharf, B. (1990). The loudness of sounds that increase and decrease continuously in level. Journal of the Acoustical Society of America, 88, 2136-2142.

Canévet, G., Scharf, B., \& Botte, M. -C. (1985). Simple and induced loudness adaptation. Audiology, 24, 430-436.

Marks, L. E., \& SLAwSON, A. W. (1966). Direct test of the power function for loudness. Science, 154, 1036-1037.

Neuhoff, J. G. (1998). Perceptual bias for rising tones. Nature, 395 , 123-124.

SCHARF, B. (1983). Loudness adaptation. In J. V. Tobias \& E. D. Schubert, Hearing research and theory (Vol. 2, pp. 1-56). Orlando, FL: Academic Press.

SCharf, B., \& Canéver, G. (1989). Loudness adaptation, loudness functions, and decruitment. ln G. Canévet, B. Scharf, A.-M. Bonnel, \& C.-A. Possamai (Eds.), Fechner Day ' 89 (pp. 137-142). Marseille: CNRS, International Society for Psychophysics.

SCHLAUCH, R.S. (1992). A cognitive influence on the loudness of tones that change continuously in level. Journal of the Acoustical Society of America, 92, 758-765.

Schlauch, R. S., \& Morison. L. M. (1995). The spectral spread of loudness adaptation in tones that decrease continuously in level. Acustica, 81, 279-280.

(Manuscript received August 11, 1998; revision accepted for publication February 26, 1999.) 